\title{
Are luminous and metal-rich Wolf-Rayet stars inflated?
}

\author{
J. Petrovic ${ }^{1,2}$, O. Pols ${ }^{2}$, and N. Langer ${ }^{2}$ \\ 1 Astronomical Institute, Radboud Universiteit Nijmegen, Toernooiveld 1, 6525 ED, Nijmegen, The Netherlands \\ e-mail: petrovic@astro.ru.nl \\ 2 Sterrenkundig Instituut, Postbus 80 000, 3508 TA Utrecht, The Netherlands
}

Received 10 December 2003 / Accepted 23 November 2005

\begin{abstract}
Aims. We investigate the influence of metallicity and stellar wind mass loss on the radius of Wolf-Rayet stars.

Methods. We have calculated chemically homogeneous models of Wolf-Rayet stars of 10 to $200 M_{\odot}$ for two metallicities $(Z=0.02$ and $Z=0.001$ ), without mass loss, using OPAL opacities. We also constructed theoretical helium main sequences for $15,18,24$ and $30 M_{\odot}$ with stellar wind mass loss rates of $10^{-5} M_{\odot} \mathrm{yr}^{-1}$ and $10^{-4} M_{\odot} \mathrm{yr}^{-1}$ and models for a $24 M_{\odot}$ star for a few intermediate values of mass loss rate. Results. We confirm the radius extension for luminous, metal-rich Wolf-Rayet stars reported previously by Ishii et al. (1999, PASJ, 51, 417), i.e. the inflation of the hydrostatic stellar radius. We also show that for small values of the stellar wind mass loss rate, an extended envelope structure is still present. However, for mass loss rates above a critical value, for which we derive an expression, Wolf-Rayet radii decrease and the stellar structure becomes compact. We briefly discuss possible evolutionary and observational consequences of an inflated envelope for a mass losing Wolf-Rayet star.
\end{abstract}

Key words. stars: fundamental parameters - stars: Wolf-Rayet - stars: interiors - stars: mass-loss - stars: winds, outflows

\section{Introduction}

Wolf-Rayet (WR) stars are the bare cores of massive stars which have lost most or all of their hydrogen-rich envelope (Chiosi \& Maeder 1986; Maeder \& Conti 1994). While in close binary systems, the loss of the envelope does not depend only on the initial stellar mass (Wellstein et al. 2001), single stars need to be more massive than $\sim 25 M_{\odot}$ to evolve into a WR star (Massey et al. 2001). WR stars are characterized by strong stellar winds, whose origin is still not well understood (Gräfener et al. 2002). In particular in hydrogen-free WR stars, which are in the focus of our study, the stellar wind is optically thick, preventing a direct determination of radii of WR stars, even when bolometric luminosities and effective temperatures are determined (Hamann et al. 1995; Moffat \& Marchenko 1996).

The knowledge of the radii of WR stars is essential for the following problems. In order to understand the strong wind of a WR star of given mass, its radius is a key quantity, as it determines the height of the potential well which has to be overcome by the wind. The winds of hydrogen-free WR stars might be explained in terms of radiative driving if the radius at the base of the wind - i.e., the radius of the sonic point - is a factor of 2...3 larger (Nugis \& Lamers 2002; Gräfener et al. 2002) than what is predicted by WR stellar structure and evolution models (Langer 1989; Mowlavi et al. 1998; Heger \& Langer 1996; Meynet \& Maeder 2003).
WR star radii are also important for the evolution of close binaries with a WR star component, in particular for initially wide binaries, where the hydrogen-rich envelope of the WR progenitor is removed in a so called common envelope evolution, resulting in a very close system (Taam \& Sandquist 2000). Interesting examples where the WR radius may have influenced the evolution of the binary systems are the massive X-ray binaries Cyg X-3 (van Kerkwijk et al. 1996) and Cyg X-1 (Brown et al. 2001).

Finally, the radii particularly of the most massive WR stars are relevant for the understanding of gamma-ray bursts (GRBs), which, according to the collapsar model (MacFadyen et al. 2001), have their origin in those WR stars which are massive enough to form a black hole at the end of their evolution (Heger et al. 2003). The prediction of this scenario that GRBs are associated with so called type Ic supernovae - which are interpreted as supernovae originating from hydrogen-free WR stars (Woosley et al. 1993, 1995) - appears to be supported by recent GRB afterglow observations (Hjorth et al. 2003).

In this paper, we investigate the radii of massive, chemically homogeneous helium star models, which have been shown to represent evolutionary models of hydrogen-free WN stars very accurately (Langer 1989). The radii of such models in the mass range $10 \ldots 30 M_{\odot}$ are usually found to be in the range $1 \ldots 2 R_{\odot}$ (Langer 1989; Schaerer \& Maeder 1992). However, while the helium star models of Ishii et al. (1999) predict radii in agreement with previous results up to $15 M_{\odot}$, 
they find a strong radius inflation for larger masses. I.e., at solar metallicity, their $30 M_{\odot}$ model has a radius of $8.6 R_{\odot}$, and at $100 M_{\odot}$ they find a helium star radius of $670 R_{\odot}$.

Here, we construct massive helium star models using OPAL opacities, which have been identified by Ishii et al. (1999) as a key model ingredient to find such large helium star radii. In Sect. 2, we describe the details of the way our models are constructed. In Sects. 3 and 4, we then describe the dependence of the radii of our helium stars on the metallicity and on the adopted stellar wind mass loss rate, respectively. Sect. 5 gives a general discussion of the effect of increased helium star radii, and Sect. 6 summarizes our results.

\section{Computational method and input physics}

We modelled the structure of chemically homogeneous zero age helium main sequence stars with the hydrodynamic stellar evolution code described in Langer et al. (1988) and Langer (1989). The OPAL opacities of Iglesias \& Rogers (1996) are implemented. We produced models at two different metallicities, $Z=0.02$ and $Z=0.001$, with an abundance distribution which is typical for hydrogen burning ashes, i.e. $X=0$ and $Y=1-Z$ and with abundances of carbon, nitrogen and oxygen equal to $2.775 \times 10^{-3} Z, 0.254 Z$ and $0.5 Z$, respectively.

Models are made for stellar masses of 10, 12, 15, 18, 24 and $30 M_{\odot}$. We constructed models without mass loss for both metallicities, and additional models with mass loss rates of $10^{-5} M_{\odot} \mathrm{yr}^{-1}$ and $10^{-4} M_{\odot} \mathrm{yr}^{-1}$ for $Z=0.02$. We also constructed a series of models for a star of $24 M_{\odot}$, with five intermediate values of the mass loss rate. Mass loss is incorporated in our models by removing a proportionate amount of mass from each shell in the outer 40 per cent of the stellar mass, while in the inner 60 percent of the stellar mass Lagrangian coordinates are used. Nuclear energy production in our models comes from the $3 \alpha$ reaction, while that of the ${ }^{14} \mathrm{~N}(\alpha, \gamma)$ reaction is neglected (see below).

The models without mass loss are set in complete hydrostatic and thermal equilibrium. In order to achieve complete relaxation, we used extremely large time steps, of the order of $10^{20}$ seconds, while preventing the consumption of nuclear fuel. These equilibrium models were also used as the initial models for the calculations with mass loss. For the models with mass loss hydrostatic equilibrium is not possible, first of all since mass loss implies non-stationarity, and second - as explained in detail in Sect. 4 - the mass loss rates considered here imply outflow velocities and, more importantly, inertia terms which are significant. However, as the mass loss time scale is longer than the dynamical time scale of the star, our mass-losing models are characterized by quasi-stationarity. We used a time step of the order of $10^{3}$ seconds to construct masslosing models, until a quasi-stationary state is achieved. In this way the amount of matter lost in the stellar wind is negligible. The change in entropy $(\mathrm{d} s / \mathrm{d} t)$ resulting from decompression due to mass loss is fully taken into account in the energy balance equation. For both types of models, the radius $R$ of our WR models is defined by an optical depth $\tau(R)=2 / 3$, as determined from a gray plane-parallel atmosphere integration.
In order to obtain an independent consistency check on our results for constant masses and on those of Ishii et al. (1999), we also computed the structure of helium star models in hydrostatic and thermal equilibrium with the stellar evolution code of Eggleton (1971) and Eggleton (1972). We used the version described by Pols et al. (1995), with the OPAL 1996 opacities implemented as described by Eldridge \& Tout (2004). Because this code code can only deal with nearly hydrostatic stars (the inertial terms are neglected) we did not consider mass loss in these calculations. The models were computed for the same two metallicities as above, on a fine grid of masses between 1 and $200 M_{\odot}$ (spaced by 0.002 in $\log M$ ). The abundance distribution is homogeneous except that ${ }^{14} \mathrm{~N}$ from the hydrogen ashes was assumed to be already consumed inside the convective core ${ }^{1}$.

\section{Results: $\dot{M}=0 ; Z=0.02$ and $Z=0.001$}

Figure 1 shows the theoretical helium zero-age main sequences as obtained from our models without mass loss, for the metallicities $Z=0.001$ and $Z=0.02$. Models with the same mass and different metallicities have roughly the same luminosity, but the effective temperature is lower for higher metal content. The influence of the metallicity becomes significant for stellar masses larger than $\sim 15 M_{\odot}$ and then increases with increasing stellar mass. For masses above $\sim 15 M_{\odot}$, the surface temperature of the metal-rich models decreases with increasing mass, i.e., the upper part of the helium ZAMS in the H-R diagram is bent towards the red.

Consequently, the helium stars more massive than $\sim 15 M_{\odot}$ with solar metallicity have significantly larger radii than the corresponding stars with a metallicity of $Z=0.001$ (cf. Table 1). For $M<15 M_{\odot}$, the radii of equal-mass models differ by less than $10 \%$. The radius of a $15 M_{\odot}$ star is $\sim 15 \%$ larger at $Z=0.02$ compared to $Z=0.001$. This difference increases with stellar mass, e.g. a $30 M_{\odot}$ star is $~ 3.6$ times larger due to the higher metal content.

The results obtained with the Langer and Eggleton codes correspond very well, as is demonstrated in Fig. 1 and Table 1. In spite of quantitative differences of order $10 \%$ between the model radii for masses larger than $24 M_{\odot}$, the qualitative behaviour is very similar and furthermore confirms the findings of Ishii et al. (1999), as can be seen by comparing our Fig. 1 with their Fig. 7. For masses greater than $100 M_{\odot}$ we find a giant structure with radii of several $100 R_{\odot}$, as did Ishii et al. This demonstrates that inflation is a common feature of massive helium star models and not the result of numerical artifacts. The models for $Z=0.001$ remain compact up to $100 M_{\odot}$, but for larger masses they are also inflated.

The inflated massive, metal-rich models have a peculiar envelope structure. Figure 2 compares the density profiles of

\footnotetext{
1 Without this assumption the main energy production comes from ${ }^{14} \mathrm{~N}(\alpha, \gamma)$ rather than the $3 \alpha$ reaction, and we find radii that are $5 . .10$ per cent larger than the results presented here. In actual WR stars nitrogen burns very quickly before any substantial $3 \alpha$ fusion has taken place, so that models in which core nitrogen is already consumed more closely represent "zero-age" helium stars.
} 
Table 1. Radii of zero age helium star models for masses in the range $10 \ldots 30 M_{\odot}$, for two metallicities, $Z=0.02$ and $Z=0.001$, as indicated by the upper index, and for various mass loss rates, i.e. $\dot{M}=0,10^{-4}, 10^{-5} M_{\odot} \mathrm{yr}^{-1}$, as indicated by the lower index. The subscript "E" refers to models calculated with the Eggleton code. The last two column lists results of Ishii et al. (1999) and Langer (1998).

\begin{tabular}{ccccccccc}
\hline$M$ & $R_{0}^{.001}$ & $R_{0, E}^{.001}$ & $R_{0}^{.02}$ & $R_{-5}^{.02}$ & $R_{-4}^{.02}$ & $R_{0, E}^{.02}$ & $R_{0, \mathrm{I}}^{.02}$ & $R_{0, \mathrm{~L}}^{.02}$ \\
$M_{\odot}$ & $R_{\odot}$ & $R_{\odot}$ & $R_{\odot}$ & $R_{\odot}$ & $\mathrm{R}_{\odot}$ & $R_{\odot}$ & $R_{\odot}$ & $R_{\odot}$ \\
\hline 10 & 0.86 & 0.87 & 0.93 & - & - & 0.93 & - & 0.92 \\
12 & 0.95 & 0.96 & 1.05 & - & - & 1.05 & 1.1 & - \\
15 & 1.08 & 1.09 & 1.26 & 1.26 & 1.16 & 1.26 & 1.3 & 1.16 \\
18 & 1.20 & 1.22 & 1.58 & 1.58 & 1.34 & 1.57 & - & - \\
24 & 1.42 & 1.43 & 3.16 & 3.11 & 1.61 & 2.85 & - & - \\
30 & 1.61 & 1.63 & 5.79 & 5.78 & 1.88 & 5.20 & 8.3 & 1.73 \\
\hline
\end{tabular}

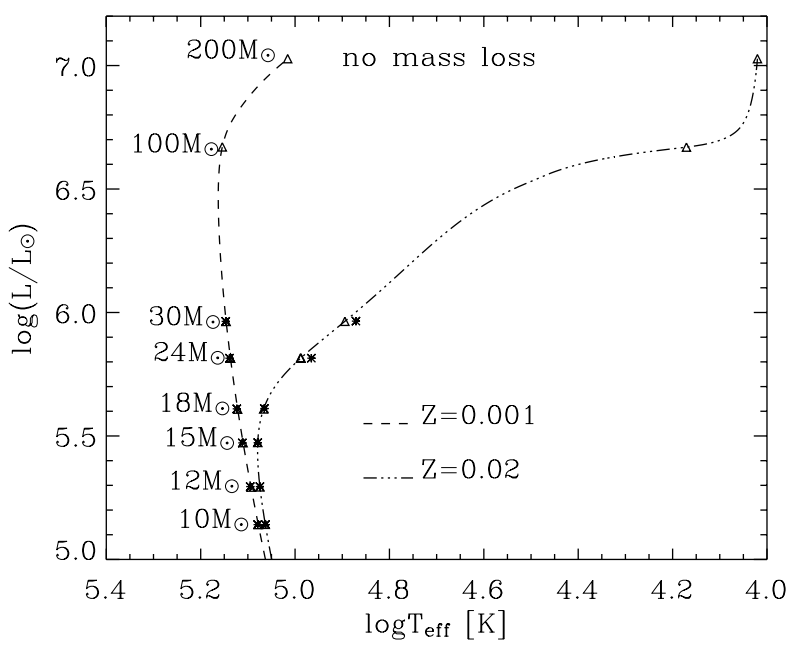

Fig. 1. Theoretical helium zero age main sequences for metallicities $Z=0.001$ and $Z=0.02$ without mass loss calculated with the Eggleton's code. Triangles indicate models for a particular mass. Stars indicate corresponding models computed with the Langer code.

our metal-rich $15 M_{\odot}$ and $24 M_{\odot}$ stars (as computed with the Langer code) with their metal-poor counterparts. An extended envelope structure and a density inversion are present in the outer layers for both masses at the higher value of the metallicity. For $15 M_{\odot}$ at $Z=0.02$, there is a narrow layer with low density $\left(\sim 10^{-9} \mathrm{~g} \mathrm{~cm}^{-3}\right)$ beneath the stellar surface, where the density reaches a value of $\sim 10^{-8} \mathrm{~g} \mathrm{~cm}^{-3}$. For the $24 M_{\odot}$ star with solar metallicity, the envelope is significantly more extended and there is a broad layer $\left(\sim 1 R_{\odot}\right)$ with a density of $\sim 10^{-10} \mathrm{~g} \mathrm{~cm}^{-3}$ beneath the surface, where the density is almost two orders of magnitude higher. The extended envelope contains only about $10^{-10}$ of the total stellar mass. Models with a metallicity of $Z=0.001$ exhibit no extended envelope structure, and the density decreases monotonously from the stellar interior to the surface.

In Figs. 3 and 4, we compare the opacity and luminosity structure of our $10 M_{\odot}$ models - for which the metal content has almost no effect on the structure - with our $24 M_{\odot}$ stars, which show a significant inflation at $Z=0.02$. The upper panel of both figures show the opacity profiles for both values of metallicity. For the model with higher metal content,

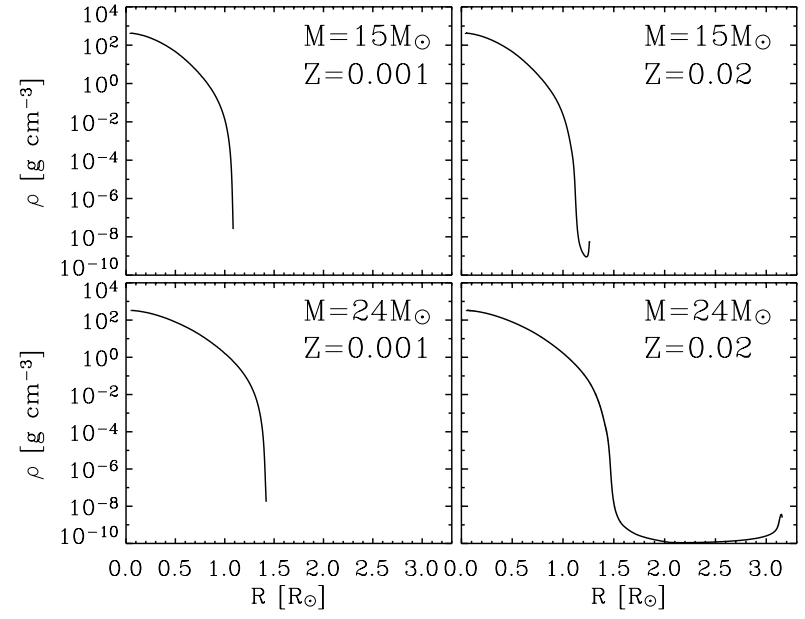

Fig. 2. Density profiles for helium stars of $15 M_{\odot}$ and $24 M_{\odot}$. Models with higher metallicity have extended envelopes and a density inversion near the surface.

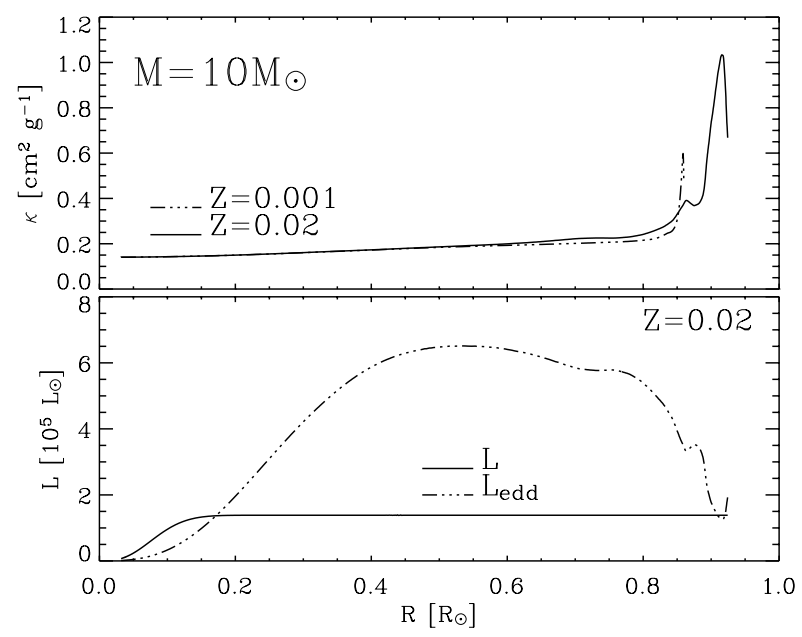

Fig. 3. Upper plot: opacity as a function of radius for $10 M_{\odot}$ helium stars of $Z=0.001$ and $Z=0.02$. Lower plot: luminosity and Eddington luminosity as a function of radius for $10 M_{\odot}$ helium star of solar metallicity.

the opacity is larger due to the iron opacity peak at $T \sim$ $2.5 \times 10^{5} \mathrm{~K}$ in the OPAL tables, which increases with metallicity. While in the $10 M_{\odot}$ models the achieved opacity is larger than at $24 M_{\odot}$, the radius range for which the opacity is high is large only in the metal-rich $24 M_{\odot}$ star.

To understand the effect of the iron opacity peak on the stellar radius, it is useful to compare the local luminosity $L$ with the local Eddington luminosity $L_{\text {Edd }}=4 \pi c G M(r) / \kappa$, where $M(r)$ is the Lagrangian mass coordinate and $\kappa$ the opacity coefficient. The lower plot of Fig. 3 shows profiles of the luminosity and the local Eddington luminosity for the metal-rich $10 M_{\odot}$ model. The Eddington luminosity is significantly higher than the local stellar luminosity throughout the star, except in a narrow convective layer close to the surface. In the $24 M_{\odot}$ model, the local stellar luminosity almost equals the Eddington luminosity in the extended outer radiative parts (between points 1 and 2 in Fig. 4), while it exceeds the Eddington value in an extended convective layer above (between points 2 and 3 ). 


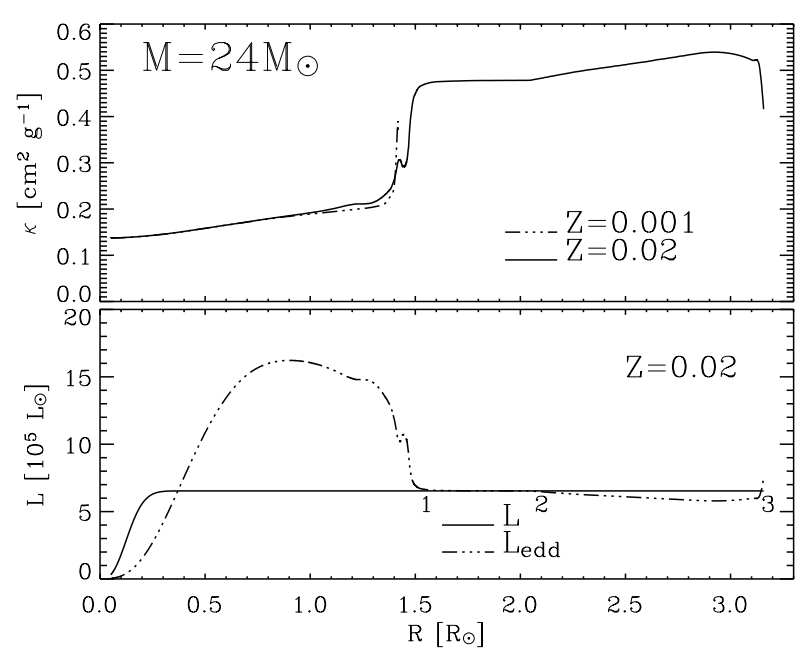

Fig. 4. Upper plot: opacity as a function of radius for a $24 M_{\odot}$ helium stars of $Z=0.001$ and $Z=0.02$. Lower plot: luminosity and Eddington luminosity as a function of radius for a $24 M_{\odot}$ helium star of solar metallicity. 1-2 Radiative layers with luminosity close to Eddington limit; 2-3 Convectively unstable layers.

We interpret this behavior as follows. The WR models considered here follow a mass-luminosity relation, approximately $L_{\star} \propto M_{\star}^{1.35}$ (Langer 1989). Therefore, for a fixed opacity, more massive stars are generally closer to their Eddington limit. Secondly, like for hydrogen-rich main sequence stars, the density of helium stars decreases with increasing stellar mass. This has the consequence that the convection zone due to the iron opacity peak - which is present in all considered models transports energy less efficiently in more massive helium stars.

Our results indicate that, while for stars below $\sim M>15 M_{\odot}$ any small super-Eddington flux can be transported by convection without structural consequences, for stars above $\sim M>$ $15 M_{\odot}$ at $Z=0.02$, a compact hydrostatic structure is impossible as it would require $L>L_{\text {Edd }}$ in part of the envelope where even convection can not carry the excess luminosity. Thus, a compact structure would give rise to an outward acceleration, i.e. to an inflation of the envelope. The reduced density due to the expansion has two consequences, a further reduced convective energy transport efficiency, and a reduction of the opacity as the iron peak decreases towards lower densities. While the first effect is limited as the convective flux in the outermost convective layers is already negligible before the expansion, the second effect allows that a hydrostatic solution is one with an extended envelope. Throughout this envelope the luminosity remains very close to the Eddington limit. At low $Z$, the envelope opacity is lower and $L_{\text {Edd }}$ larger, so that a compact hydrostatic structure remains possible up to a larger mass. Hence, the increase of the envelope opacity with $Z$, and the increase in $L / M$ with mass, are the prime causes for the extended envelopes and large radii of massive high-metallicity WR stars.

\section{Results: $\dot{M}>0 ; Z=0.02$}

In this section, we investigate the influence of stellar wind mass loss on the extended envelope structure obtained for massive, metal-rich hydrostatic helium star models. For $Z=0.02$, we calculated hydrodynamic, mass losing models for 15,18 ,

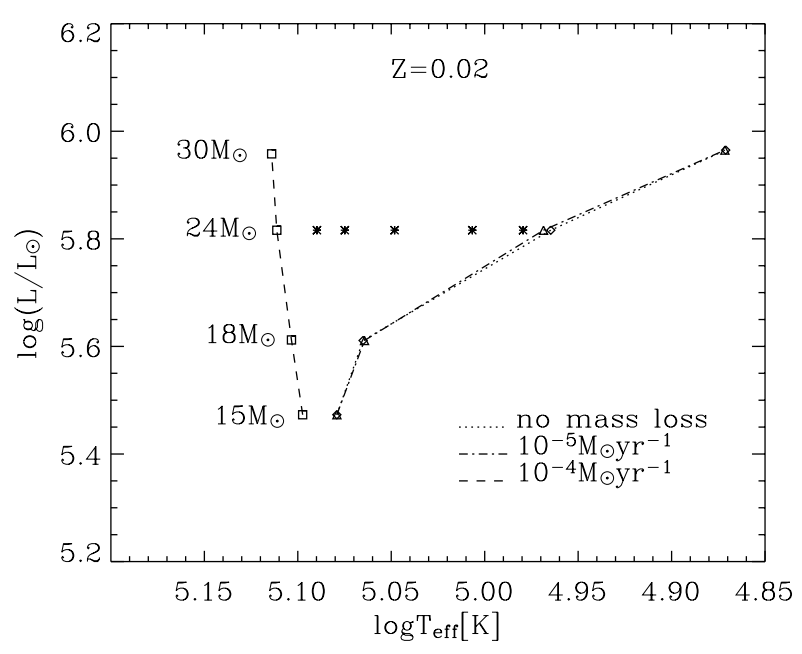

Fig. 5. Theoretical helium zero age main sequences for mass loss rates $0,10^{-5} M_{\odot} \mathrm{yr}^{-1}$ and $10^{-4} M_{\odot} \mathrm{yr}^{-1}$. Models have been calculated for 15 , 18, 24 and $30 M_{\odot}$. Star symbols indicate $24 M_{\odot}$ models for intermediate mass loss rates (see Fig. 6).

24 and $30 M_{\odot}$ for two different mass loss rates: $10^{-5} M_{\odot} \mathrm{yr}^{-1}$ and $10^{-4} M_{\odot} \mathrm{yr}^{-1}$. For $24 M_{\odot}$, we constructed additional models for intermediate mass loss rates. Figure 5 compares the location of these models in the HR-diagram to the location of the models without mass loss.

We notice that a mass loss rate of $10^{-5} M_{\odot} \mathrm{yr}^{-1}$ has almost no effect on the positions of the stars in the H-R diagram. The envelopes of stars more massive than $\sim 15 M_{\odot}$ are still extended and their effective temperatures are relatively low. However, a mass loss rate of $10^{-4} M_{\odot} \mathrm{yr}^{-1}$ significantly changes the envelope structure: The stars are compact with high effective temperatures, and indeed follow closely the mass-radius relation for helium stars of Langer (1989). For $24 M_{\odot}$, we have computed models with intermediate mass loss rates, whose radii are plotted in Fig. 6. It shows that the radii of our models are not bimodal, as might be suggested by Fig. 5, but change continuously and monotonously as a function of the adopted mass loss rate.

Figure 7 shows the density profiles of a $24 M_{\odot}$ star for four different mass loss rates. With a mass loss rate of $2.5 \times$ $10^{-5} M_{\odot} \mathrm{yr}^{-1}$, the radius is smaller than that of a constant-mass model. With even higher mass loss rates the envelope becomes more and more compact. On the other hand, the structure in the core is unaffected by mass loss: the density profiles for $R<1.5 R_{\odot}$ are virtually identical for each adopted mass loss rate. The amount of mass lost at this point is negligible, and even for a mass-loss rate of $10^{-4} M_{\odot} \mathrm{yr}^{-1}$ the integrated gravitational energy amounts to only a small (negative) fraction (4 per cent) of the stellar luminosity. The core remains in hydrostatic equilibrium, unlike the extended envelope, as we discuss below.

Figure 8 shows the velocity profiles in the outer stellar layers for our $24 M_{\odot}$ star for three different values of the mass loss rate, as calculated with the hydrodynamic evolution code. The stellar mass loss implies an outward mass flux through the 


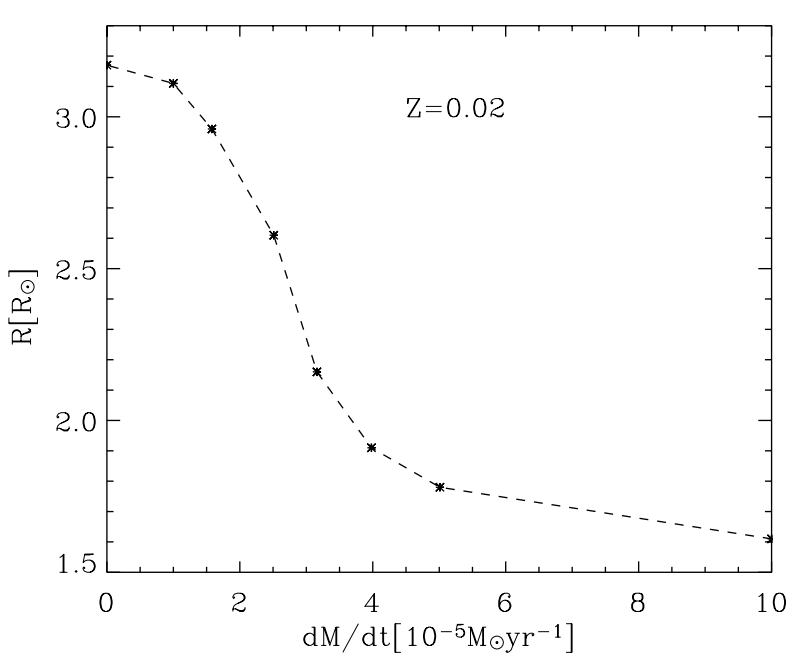

Fig. 6. Radii of eight $24 M_{\odot}$ helium star models at $Z=0.02$ as a function of the adopted mass loss rate. These models are also indicated in the HR diagram (Fig. 5).

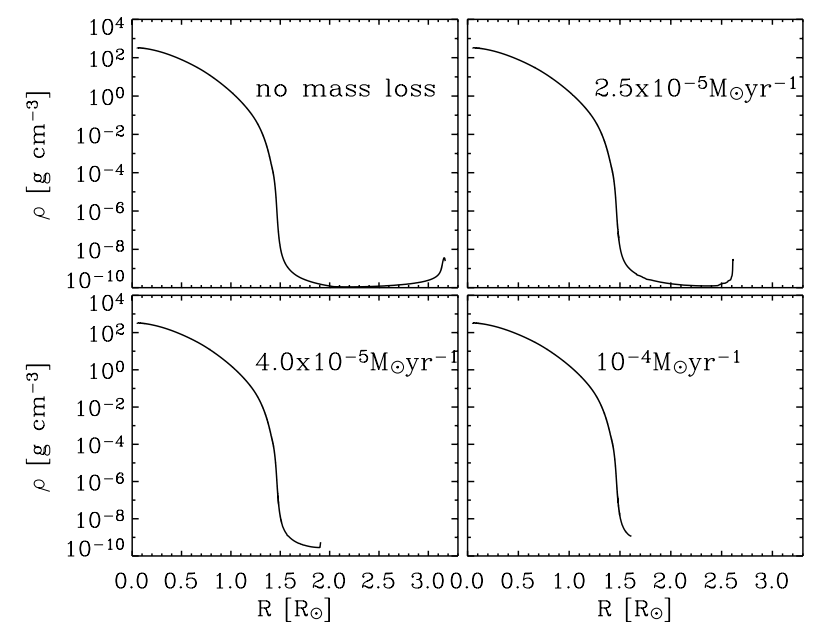

Fig. 7. Density profiles for helium star models of $24 M_{\odot}$ for four different values of the mass loss rate: $0,2.5 \times 10^{-5} M_{\odot} \mathrm{yr}^{-1}, 4.0 \times$ $10^{-5} M_{\odot} \mathrm{yr}^{-1}, 10^{-4} M_{\odot} \mathrm{yr}^{-1}$.

envelope. Mass conservation and quasi-stationarity imposes that the lower the density the faster the matter has to move:

$v=\dot{M} /\left(4 \pi r^{2} \rho\right)$.

For the adopted mass loss rates, the low densities in the extended envelopes of our models $\left(\sim 10^{-10} \mathrm{~g} \mathrm{~cm}^{-3}\right.$; cf. Fig. 7) imply velocities of several hundred kilometers per second. This can be compared to the isothermal sound speed in the stellar envelope,

$v_{\mathrm{s}}=\sqrt{R T / \mu}$,

where $T$ is the temperature, $R$ is the gas constant and $\mu$ is the mean molecular weight. Both are plotted in Fig. 8, which shows that the implied flow velocities in the extended envelopes of our models are highly supersonic.

In the hydrostatic model, the acceleration due to the pressure gradient is balanced by gravity. In the models with mass loss, the sum of these two accelerations is not zero. Instead,

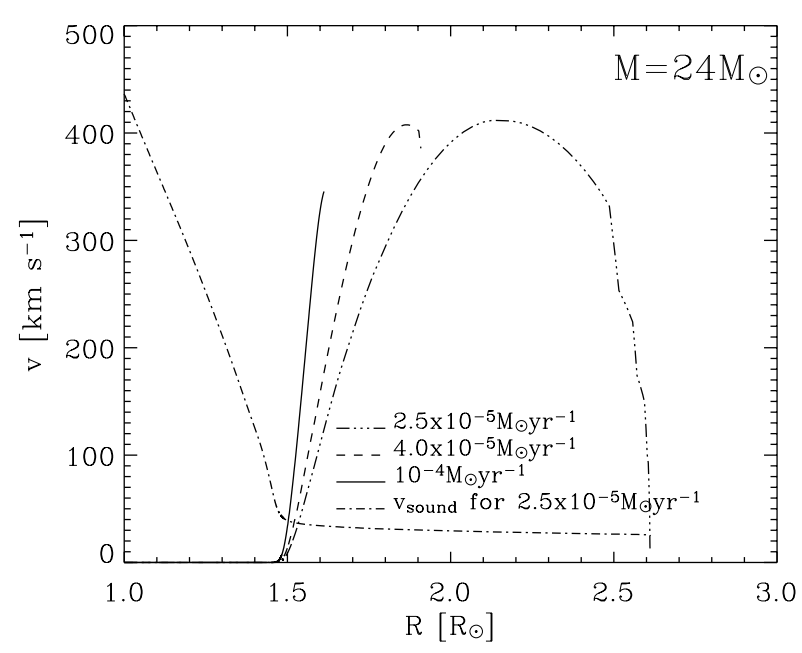

Fig. 8. Velocity profiles for $24 M_{\odot}$ helium star models calculated using the hydrodynamic evolution code for three different mass loss rates: $2.5 \times 10^{-5} M_{\odot} \mathrm{yr}^{-1}, 4.0 \times 10^{-5} M_{\odot} \mathrm{yr}^{-1}, 10^{-4} M_{\odot} \mathrm{yr}^{-1}$, compared with the sound speed for $2.5 \times 10^{-5} M_{\odot} \mathrm{yr}^{-1}$.

there is an accelerated radial motion and the inertial acceleration becomes important, and more so for larger mass loss rates:

$\frac{\partial p}{\partial r}=-\frac{G m}{r^{2}} \rho-\frac{\partial^{2} r}{\partial t^{2}} \rho$

where $p$ is the pressure, $r$ the radial coordinate, $G$ the gravitational constant and $\rho$ is the density.

We can see from Eq. (3) that if the inertia term increases and becomes comparable to the gravitational acceleration, the absolute value of the pressure gradient also increases, which means that the pressure decreases faster towards the surface and the resulting stellar radius is smaller. For a mass loss rate of $\sim 10^{-5} M_{\odot} \mathrm{yr}^{-1}$, the inertia term is still almost negligible, but it is of the order of the gravitational acceleration in the outer envelope for $\dot{M}=10^{-4} M_{\odot} \mathrm{yr}^{-1}$. This explains why the envelope structure is not affected for the lower mass loss rates considered here.

The critical mass loss rate above which the envelope structure will be affected can be estimated as follows. Approximating the inertia term as $\frac{\mathrm{d} v}{\mathrm{~d} t}=v \frac{\mathrm{d} v}{\mathrm{~d} r} \simeq \frac{v^{2}}{R_{\star}}$, and requiring it to be smaller than the gravitational acceleration, $\frac{G M_{\star}}{R_{\star}^{2}}$, constrains the outflow velocity $v$ to $v<\sqrt{\frac{G M_{\star}}{R_{\star}}}$, i.e., to values below the escape velocity. For a given density $\rho$ in the region of the density inversion this constrains the mass loss rate to

$\dot{M}<4 \pi R_{\star}^{2} \rho \sqrt{\frac{G M_{\star}}{R_{\star}}}$

for an extended envelope structure not to be much affected by mass loss. Since the largest inertial acceleration occurs in the lowest-density regions of the envelope, one should use the minimum envelope density in Eq. (4). For $R_{\star}=1.5 R_{\odot}$, $M_{\star}=24 M_{\odot}$ and $\rho=10^{-10} \mathrm{~g} \mathrm{~cm}^{-3}$ (from Fig. 7), we get $\dot{M}<4 \times 10^{-5} M_{\odot} \mathrm{yr}^{-1}$. 


\section{Discussion}

We confirm the findings of Ishii et al. that hydrostatic models of massive and metal-rich helium stars are inflated when mass loss is neglected. In particular, we find the bending of the helium star ZAMS $(Z=0.02)$ towards cooler surface temperatures to occur at the same mass $\left(15 M_{\odot}\right)$ as Ishii et al. At lower metallicity $(Z=0.001)$ bending also occurs, but at much higher mass $\left(100 M_{\odot}\right)$.

This ZAMS bending, which is clearly connected with the inflation phenomenon, has indeed been found earlier. It was first reported by Kato $(1985,1986)$ for supermassive stars $\left(\sim 10^{6} M_{\odot}\right)$ with Compton-scattering opacity. Glatzel et al. (1993), using OPAL opacities, also noted this phenomenon in their helium star models. Langer (1989) calculated models of chemically uniform hydrogen-free core helium burning stars using Los Alamos radiative opacities. His helium ZAMS for $Z=0.02$ indicated bending at about $60 M_{\odot}$, which shows that the inflation phenomenon does not depend only on the iron peak in the OPAL opacities, but that it may also occur for smaller envelope opacities, though only for stars with a larger luminosity-to-mass ratio. The chemically homogeneous, extremely CO-enriched models of Langer (1989) - corresponding to the helium burning terminal age main sequence - showed a bending around $15 M_{\odot}$, likely due to the increase of the Los Alamos opacities with carbon and oxygen abundance. We note further that the inflation phenomenon is not restricted to hydrogen-free stars but occurs also for normal metal-rich ZAMS stars (Schaerer et al. 1993; Glatzel \& Kiriakidis 1993; Mowlavi et al. 1998; Stothers \& Chin 1999; Ishii et al. 1999).

However, the mass loss rates of luminous, hydrogen-free stars are very high, of the order of $10^{-5} \ldots 10^{-4} M_{\odot} \mathrm{yr}^{-1}$ for the helium stars in mass range considered here (Nugis \& Lamers 2000). In Sect. 4 we showed that mass loss with rates above a critical level (Eq. 4) leads to a reduction or even vanishing of the envelope extension. It is therefore doubtful if, for the case of hydrogen-free stars, the effect of inflation is ever realized in nature. One might argue that an increased metallicity leads to a lower critical mass for the onset of inflation in models without mass loss. But on the other hand, a higher metallicity may also lead to increased mass loss rates (Crowther et al. 2002) which would counteract the effect of a lower critical mass for inflation.

One may also speculate on the effect that the inflation may have on the evolution of the star. Models with an inflated envelope have a very peculiar structure: a hot stellar core covered by an extended, very dilute and almost transparent region which is covered by a rather thin and relatively dense shell. One may wonder about the stability of such a configuration. Glatzel et al. (1993) found that such objects are pulsationally unstable, i.e. even without mass loss, large scale velocity fields may be induced. On the other hand, even moderate radiation-induced outflows lead to large velocity gradients due to the low densities obtained in the extended envelopes. This may give rise to large accelerations due to line driving in the CAK model (Castor et al. 1975), which is proportional to the velocity gradient. I.e., an extended envelope may lead to an increased mass loss, which steepens the velocity gradient (Fig. 8) and may enhance the mass loss further. This way, a mass loss instability may be induced. Consequently, the evolutionary time scale of inflated stars may be very short, which could correspond to the fact that solar-metallicity hydrogen-rich and hydrogenfree stars with masses above the predicted inflation limit (i.e., $\sim 120 M_{\odot}$, Glatzel \& Kiriakidis (1993), and $\sim 15 M_{\odot}$, Ishii et al. (1999), respectively) are practically absent in our Galaxy.

\section{Conclusions}

Solar metallicity helium stars above $\sim 15 M_{\odot}$ develop an extended envelope structure if mass loss is neglected. Consequently, their effective temperature decreases and the helium ZAMS bends towards the red in the H-R diagram. At low metallicity this feature does not occur or is shifted to much higher masses.

The occurrence of inflated envelopes is related to the proximity of the stellar luminosity to its effective Eddington luminosity, i.e. to a high luminosity-to-mass ratio or a high envelope opacity. I.e., massive and metal-rich stars are prone to inflation. While the iron opacity peak in the OPAL tables greatly increases the occurrence of inflated envelopes, the phenomenon itself is not dependent only on it.

However, we showed that even in massive, metal-rich stars, inflation is avoided if the stellar wind mass loss rate exceeds a critical value (Eq. (4)). Observationally derived mass loss rates of WR stars are at or above the critical value. Furthermore, as the surfaces of WR stars are not directly observable, it remains possible that inflated envelopes are not present in any of them. On the other hand, inflated envelopes may be unstable and lead to enhanced mass loss. It is remarkable in this context that the critical WR mass loss rate for inflation is close to the observed one (Nugis \& Lamers 2000), and that few hydrogen-free stars, if any, can be found above the critical mass for inflation, which is about $15 M_{\odot}$ for solar metallicity.

\section{References}

Brown, G. E., Heger, A., Langer, N., et al. 2001, New Astronomy, 6, 457

Castor, J. I., Abbott, D. C., \& Klein, R. I. 1975, ApJ, 195, 157

Chiosi, C., \& Maeder, A. 1986, ARA\&A, 24, 329

Crowther, P. A., Dessart, L., Hillier, D. J., Abbott, J. B., \& Fullerton, A. W. 2002, A\&A, 392, 653

Eggleton, P. P. 1971, MNRAS, 151, 351

Eggleton, P. P. 1972, MNRAS, 156, 361

Eldridge, J. J., \& Tout, C. A. 2004, MNRAS, 348, 201

Glatzel, W., \& Kiriakidis, M. 1993, MNRAS, 262, 85

Glatzel, W., Kiriakidis, M., \& Fricke, K. J. 1993, MNRAS, 262, L7

Gräfener, G., Koesterke, L., \& Hamann, W.-R. 2002, A\&A, 387, 244

Hamann, W.-R., Koesterke, L., \& Wessolowski, U. 1995, A\&A, 299, 151

Heger, A., \& Langer, N. 1996, A\&A, 315, 421

Heger, A., Fryer, C. L., Woosley, S. E., Langer, N., \& Hartmann, D. H. 2003, ApJ, 591, 288

Hjorth, J., Sollerman, J., Møller, P., et al. 2003, Nature, 423, 847

Iglesias, C. A., \& Rogers, F. J. 1996, ApJ, 464, 943

Ishii, M., Ueno, M., \& Kato, M. 1999, PASJ, 51, 417

Kato, M. 1985, PASJ, 37, 311

Kato, M. 1986, Ap\&SS, 119, 57 
Langer, N. 1989, A\&A, 210, 93

Langer, N. 1998, A\&A, 329, 551

Langer, N., Kiriakidis, M., El Eid, M. F., Fricke, K. J., \& Weiss, A. 1988, A\&A, 192, 177

MacFadyen, A. I., Woosley, S. E., \& Heger, A. 2001, ApJ, 550, 410

Maeder, A., \& Conti, P. S. 1994, ARA\&A, 32, 227

Massey, P., DeGioia-Eastwood, K., \& Waterhouse, E. 2001, AJ, 121, 1050

Meynet, G., \& Maeder, A. 2003, A\&A, 404, 975

Moffat, A. F. J., \& Marchenko, S. V. 1996, A\&A, 305, L29

Mowlavi, N., Schaerer, D., Meynet, G., et al. 1998, A\&AS, 128, 471

Nugis, T., \& Lamers, H. J. G. L. M. 2000, A\&A, 360, 227

Nugis, T., \& Lamers, H. J. G. L. M. 2002, A\&A, 389, 162
Pols, O. R., Tout, C. A., Eggleton, P. P., \& Han, Z. 1995, MNRAS, 274, 964

Schaerer, D., \& Maeder, A. 1992, A\&A, 263, 129

Schaerer, D., Charbonnel, C., Meynet, G., Maeder, A., \& Schaller, G. 1993, A\&AS, 102, 339

Stothers, R. B., \& Chin, C. 1999, ApJ, 522, 960

Taam, R. E., \& Sandquist, E. L. 2000, ARA\&A, 38, 113

van Kerkwijk, M. H., Geballe, T. R., King, D. L., van der Klis, M., \& van Paradijs, J. 1996, A\&A, 314, 521

Wellstein, S., Langer, N., \& Braun, H. 2001, A\&A, 369, 939

Woosley, S. E., Langer, N., \& Weaver, T. A. 1993, ApJ, 411, 823

Woosley, S. E., Langer, N., \& Weaver, T. A. 1995, ApJ, 448, 315 